Article

\title{
Multi-Format Wavelength Conversion Using Quantum Dash Mode-Locked Laser Pumps
}

\author{
Yousra Ben M'Sallem, Chul Soo Park, Sophie LaRochelle and Leslie A. Rusch * \\ Department of Electrical and Computer Engineering, Center for Optics, Photonics and Lasers, \\ Université Laval, Québec G1S 3A8, QC, Canada; E-Mails: yousra.ben-msallem.1@ulaval.ca (Y.B.M.); \\ chul-soo.park@gel.ulaval.ca (C.S.P.); Sophie.Larochelle@gel.ulaval.ca (S.L.)
}

* Author to whom correspondence should be addressed; E-Mail: rusch@gel.ulaval.ca; Tel.: +1-418-656-2131; Fax: +1-418-656-3159.

Received: 21 March 2015 / Accepted: 30 April 2015 / Published: 14 May 2015

\begin{abstract}
We investigate and compare the performance of wavelength conversion for two different non-return-to-zero (NRZ) modulation formats at $40 \mathrm{~Gb} / \mathrm{s}$ : on off keying (OOK) and differential phase-shift keying (DPSK). To achieve wide wavelength coverage and integrability, we use a dual pump scheme exploiting four-wave mixing in semiconductor optical amplifiers. For phase stability, we use a quantum-dash mode-locked laser (QD-MLL) as a multi-wavelength source for the dual pumps, with tunability provided by the output filter. The significant sidelobes of the DPSK spectrum (relative to OOK) require the balancing of the pump proximity to the original signal (facilitating high conversion efficiency) with the signal degradation from the pump spectrum overlapping the converted DPSK signal. We achieve a conversion efficiency near $-3.6 \mathrm{~dB}$ for OOK and $-5.4 \mathrm{~dB}$ for DPSK across a $12 \mathrm{~nm}$ tuning range with low input powers $(1 \mathrm{dBm})$. We measure bit error rate (BER) and obtain error free transmission (BER $<10^{-9}$ ) with a power penalty less than $2 \mathrm{~dB}$ for OOK and $3 \mathrm{~dB}$ for DPSK.
\end{abstract}

Keywords: four wave mixing (FWM); mode-locked lasers; optical networks; packet switching; quantum-dash mode-locked laser (QD-MLL); wavelength converter 


\section{Introduction}

Future optical networks will adopt advanced modulation formats to increase bit rate and improve spectral efficiency [1]. The eventual introduction of coherent detection for reception will also facilitate innovations such as filterless networks. Adding wavelength conversion to the mix achieves the most flexible, dynamically-reconfigurable network [2-4]. Wavelength converters must be able to support both legacy intensity modulation systems and more challenging formats incorporating phase modulation.

Several wavelength conversion technologies have been proposed; however, semiconductor optical amplifiers (SOAs) offer many advantages over other solutions, such as ultra-fast response time, cost-effectiveness, compactness and the possibility of photonic integration [5]. Four-wave mixing (FWM) in SOAs for wavelength conversion preserves both the signal phase and amplitude, hence offering compatibility across modulation formats, as well as supporting high bit rates [6-8]. The use of dual pumps provides wide tunability $[9,10]$. For example, a $100 \mathrm{~nm}$ tuning range was achieved in [11] with a quantum dot semiconductor optical amplifier.

We recently proposed a wavelength conversion scheme exploiting a quantum-dash mode-locked laser (QD-MLL) combined with a frequency selective filter to provide two tunable wavelength pumps for FWM in SOAs. This scheme offers wide wavelength coverage for conversion; it is tunable, modulation-format-independent and provides stable phase. Our previous experiment [12] demonstrated wavelength conversion of $10 \mathrm{~Gb} / \mathrm{s}$ on-off keying (OOK) based on FWM in SOAs. We did not assess the conversion efficiency of our technique for phase modulated sources.

Tunability of wavelength converters is typically predicated upon tunable laser sources. Support of phase modulated signals with such sources can lead to elevated phase noise levels until the laser stabilizes following tuning [13-15]. The use of a multi-wavelength source based on QD-MLLs, combined with a tunable filter to select wavelengths, avoids this transient increase in laser phase noise. QD-MLLs are multi-wavelength sources that exhibit low-noise, high thermal stability, narrow linewidth, broad gain spectrum and reasonable output power [16]. The optical spectrum of the QD-MLLs presents multiple modes with spacing of $100 \mathrm{GHz}$, thus providing a gamut of wavelengths for the two pumps. Instead of two tunable lasers for pumps for wide spectral coverage, we proposed [12] the use of one multi-wavelength source and two tunable filters. As all concerned devices (tunable lasers, SOAs, tunable filters and QD-MLLs) can be integrated, we assume they are equal cost/complexity solutions.

In this paper we examine intensity modulated, OOK and differential phase shift keying (DPSK) signals. DPSK exhibits various advantages over conventional OOK, such as better receiver sensitivity, improved tolerance to chromatic dispersion, and robustness to optical filtering and fiber impairments [1,17-19]. We previously demonstrated high conversion efficiency of OOK at low pump powers when adopting a strategy of locating one pump as close as possible to the input signal while avoiding filtering effects on the converted signal. The significant sidelobes of the DPSK spectrum (relative to OOK), are aggravated during FWM and post-filtering, leading us to identify a modest penalty in bit error rate (BER) performance for DPSK. While DPSK was examined in [8] for a cascade, wide tunability and direct comparison of OOK and DPSK were not investigated.

We demonstrate wavelength conversion at $40 \mathrm{~Gb} / \mathrm{s}$ based on FWM in an extremely nonlinear SOA (XN-SOA) with a QD-MLL as a dual pump source for OOK and DPSK signals. Wavelength conversion 
is examined from a fixed input signal to a set of 16 output wavelengths with $100 \mathrm{GHz}$ spacing, for a conversion range of $12 \mathrm{~nm}$. Error-free transmission (BER below $10^{-9}$ ) with power penalty less than $2 \mathrm{~dB}$ for OOK and less than $3 \mathrm{~dB}$ for DPSK is observed. We obtain typical conversion efficiency of $-3.6 \mathrm{~dB}$ for OOK and $-5.4 \mathrm{~dB}$ for DPSK. Those values of conversion efficiency outperform previous reported results while requiring much lower pump powers $[8,10]$. In [10] many compromises were made to achieve polarization independence, at the cost of a bit error floor and extremely limited tuning range.

We establish the viability of our proposal to obtain good phase noise performance with QD-MLL pumps in an architecture that assures phase stability during tuning. In addition, the spectral efficiency we report is higher than previous reports in the literature, when combined with a wide tuning range and moderate pump power. We also highlight the particular challenges in achieving this performance when passing from OOK to DPSK modulation. This paper is organized as follows. In Section 2, we review the structure and principle of operation of the proposed converter. In Section 3, we present the experimental setup. Experimental demonstrations and results are provided. We conclude the paper in Section 4.

\section{Tunable Wavelength Converter Scheme}

In this section we recap the structure and mode of operation of the proposed wavelength converter; a detailed description is available in [12]. As illustrated in Figure 1a, the signal is combined with two pumps and input to a SOA optimized for nonlinear operation. The output of the SOA is filtered to choose the FWM product at the desired wavelength. The two pumps are supplied by a QD-MLL filtered by two bandpass tunable optical filters. Our experimentation examines the static performance of wavelength conversion, hence filters are tuned manually.

The QD-MLL laser structure is grown by gas source molecular beam epitaxy on S-doped (100) InP wafer. The active area consists of 6 InAs-based QD layers. QD-MLLs provide multi-wavelength sources that have low-noise, high thermal stability, narrow linewidth $(10 \mathrm{MHz})$, broad gain spectrum, reasonable output power and cost [16]. QD-MLLs can operate in active mode-locking from $10 \mathrm{GHz}$ to $20 \mathrm{GHz}$ and $40 \mathrm{GHz}$ and at higher repetition rates (above $100 \mathrm{GHz}$ ) in passive mode locking. One of the most interesting features of QD-MLL lasers is that passive mode-locking can be easily achieved without any saturable absorber. The cavity length of the QD-MLL defines the channel spacing. To achieve 100-GHz channel spacing for WDM comb generation, a cavity length of $420 \mu \mathrm{m}$ was used as in our experiment.

Figure $1 \mathrm{~b}$ depicts the measured optical spectrum for the laser operated in the passive mode-locking or free-running condition; the applied current to the active region was $250 \mathrm{~mA}$. Due to mode-locking, a flat and wide spectrum centered at $1550 \mathrm{~nm}$ is observed. Sixteen optical modes are available within the $3 \mathrm{~dB}$ optical bandwidth of $12.8 \mathrm{~nm}(1549.9 \mathrm{~nm}$ to $1562.7 \mathrm{~nm})$ and can be used as pumps.

An exhaustive review of the characteristics of the QD-MLL is available in [16], including its applicability to all-optical clock recovery, millimeter wave generation, and WDM transmission. The linewidth of the QD-MLL is an important parameter for advanced modulation format systems. The QD-MLL linewidth of the laser we used was measured using a self-heterodyne technique [20]. The linewidth of each optical mode of the QD-MLL is approximately $10 \mathrm{MHz}$. In our experiments the linewidth of the original, unconverted signals is $100 \mathrm{kHz}$. We did not observe any significant spectral broadening after wavelength conversion. 


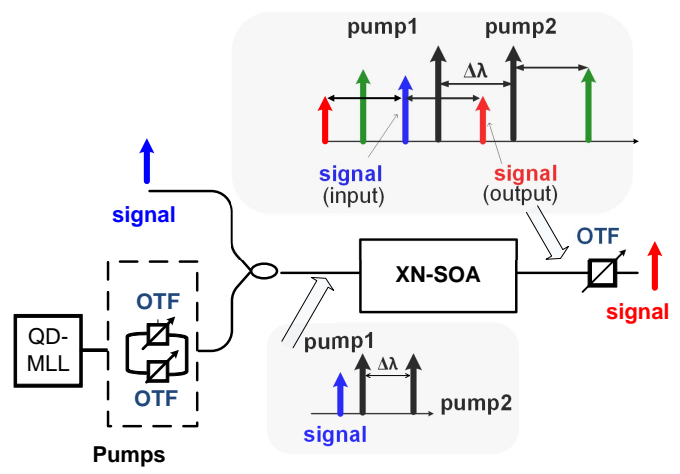

(a)

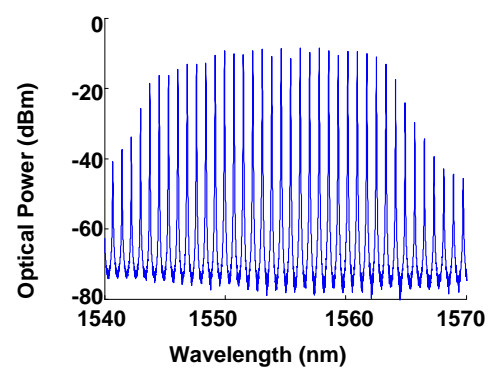

(b)

Figure 1. (a) Tunable wavelength converter, (b) quantum-dash mode-locked laser (QD-MLL) spectrum.

For a parallel polarization dual-pump configuration, the two pumps and signal are aligned in polarization before entering the SOA. As seen in the cartoon spectra in Figure 1a, nonlinear beating generates two copies of the signal (without phase conjugation) having the same polarization state as the signal with a precise conversion detuning [9]; the lower wavelength copy in red will be filtered out, while the upper wavelength copy in red will be retained as the output converted wavelength. The conversion detuning is equal to the pump detuning $\left(\Delta \lambda=\left|\lambda_{\text {pump }_{1}}-\lambda_{\text {pump }_{2}}\right|\right)$; illustrated with thin horizontal black lines with double arrows in Figure 1a. We define the conversion range as the maximumFpola achievable conversion detuning. To maximize conversion efficiency the first pump is set as close to the signal as possible, while the second pump is swept allowing wavelength conversion of the signal over a wide conversion range.

While all signals are maintained at the same optical power level at the input of the XN-SOA, the powers of the converted signals differ at the output because of the FWM efficiency fluctuation. Different experimental demonstrations for wavelength conversion with dual pumps having polarizations aligned with the input signal in non-linear SOAs appear in the literature [10,21-23], reporting conversion efficiency as a function of the small signal gain of the SOA, the SOA polarization sensitivity, the input signal powers and the detuning between the pump and the signal. We select power levels and adequate spacing between the signal and first pump that provide very good FWM efficiency and OSNR level as discussed in the next sections. 


\section{Experimental Demonstration}

In this section, we investigate the use of one QD-MLL as a multi-wavelength laser pump to generate FWM products in XN-SOA (CIP XN OEC 1550). In [12], we reported a comparison of the performance of the XN-SOA with two other commercial SOAs (CIP NL OEC 1550 and Covega 1117 SOA). The $\mathrm{XN}-\mathrm{SOA}$ greatly outperformed the other two models examined. The higher gain and saturation power leads to the XN-SOA offering the best FWM efficiency [24], and it is our choice for experiments reported here. We compare the performance of the proposed wavelength converter for OOK and DPSK. We demonstrate wavelength conversion over a $12 \mathrm{~nm}$ conversion range and BER measurements for 16 channels. Conversion efficiency, optical signal-to-noise ratio (OSNR) and power penalty for both modulation formats are reported and compared.

\subsection{Experimental Setup}

Figure 2 gives the experimental setup of a $40 \mathrm{~Gb} / \mathrm{s}$ non-return-to-zero (NRZ) system. The transmitter is comprised of a $40 \mathrm{~Gb} / \mathrm{s}$ pattern generator that generates a length $2^{31}-1$ pseudo random bit sequence, an external cavity laser source with $100 \mathrm{kHz}$ linewidth. The $40 \mathrm{~Gb} / \mathrm{s} \mathrm{LiNbO}_{3} \mathrm{Mach}-\mathrm{Zehnder}$ external modulator generates NRZ-OOK and NRZ-DPSK data by changing the bias point and the data voltage. The optical bandpass filter ( Filter $_{5}, 0.7 \mathrm{~nm}$ ) is used to reduce amplified spontaneous emission (ASE) noise.

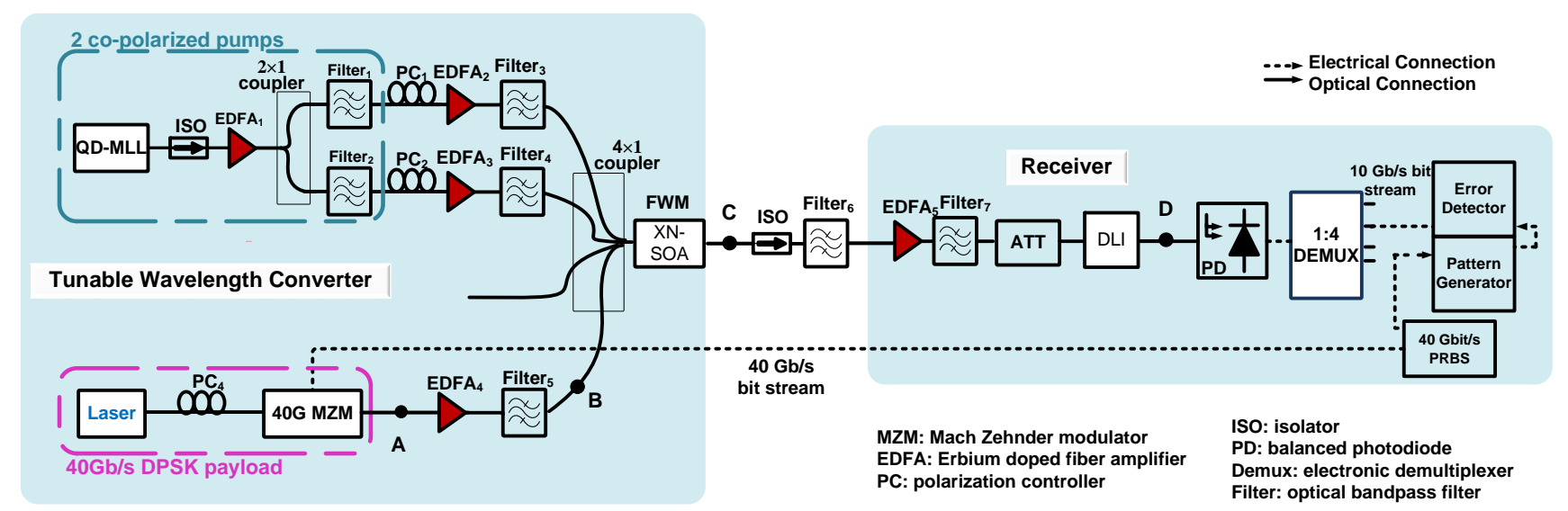

Figure 2. Setup for wavelength conversion of $40 \mathrm{Gbit} / \mathrm{s}$ on off keying (OOK) and differential phase-shift keying (DPSK) signals.

The output spectrum of the QD-MLL is split by a $1 \times 2$ coupler, amplified and then filtered (Filter ${ }_{1}$, Filter $_{2}, 0.25 \mathrm{~nm}$ ) to select two modes of the QD-MLL, forming pumps P1 and P2. ASE is suppressed by Filter ${ }_{3}$ and Filter $_{4}(0.7 \mathrm{~nm})$. The two pumps are polarization aligned with the $40 \mathrm{~Gb} / \mathrm{s}$ modulated data and all three signals are injected into the XN-SOA using a 14 coupler.

The XN-SOA used for FWM has a small signal gain of $33 \mathrm{~dB}$, gain variation $<1 \mathrm{~dB}$ with input polarization, and $15 \mathrm{dBm}$ output saturation power at $550 \mathrm{~mA}$ bias current. At the output of the XN-SOA, the converted signal is selected using a manually tuned optical bandpass filter ( Filter $_{6}, 0.25 \mathrm{~nm}$ ). The 3-dB bandwidth of the filter is narrower than the signal bandwidth, but as the filter has slow roll-off, performance is little impacted. Optical isolators are used to prevent reflections. 
Our receiver is composed of an erbium doped fiber amplifier (EDFA) to amplify the selected signal, an optical bandpass filter to reduce ASE noise and a variable optical attenuator to adjust the received optical power. A delay-interferometer (DLI) converts the phase modulation into intensity modulation when using DPSK. A 1:4 electronic demultiplexing stage after photodetection is used to demultiplex the $40 \mathrm{~Gb} / \mathrm{s}$ bit stream down to a rate compatible with our BER analyzer.

We measure OSNR, conversion efficiency, and BER over a wide tuning range by fixing the input signal and first pump wavelengths, and sweeping the second pump over the 16 available wavelengths. We measure the conversion efficiencies for three values of input signal and first pump wavelengths while again sweeping the second pump over the 16 available wavelengths. This gives us the possibility to vary the pump detuning value from $0.8 \mathrm{~nm}$ to $12 \mathrm{~nm}$ for OOK, and $1.6 \mathrm{~nm}$ to $12.8 \mathrm{~nm}$ for DPSK.

\subsection{Experimental Results}

Our figure of merit is conversion efficiency, defined here as the ratio between the power of the converted signal at the output of the XN-SOA (measured at point $\mathrm{C}$ of Figure 2) and the input power of the original signal (measured at point B). In our experiment, the power of the pumps is set to $1 \mathrm{dBm}$, while the power of signal is set to $-0.5 \mathrm{dBm}$ and $2.5 \mathrm{dBm}$ for OOK and DPSK modulation, respectively. As the DPSK signal is constant envelope, this equates to equal peak power for OOK and DPSK. This operating point was found to yield optimal conversion efficiency for OOK in [12].

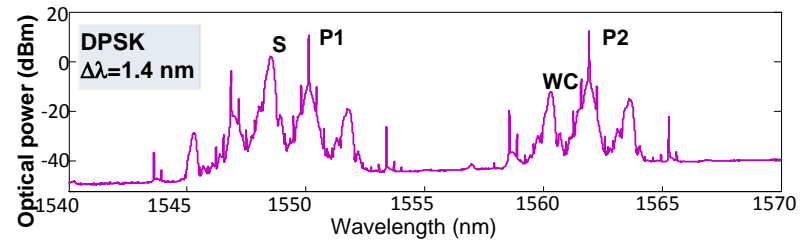

(a)

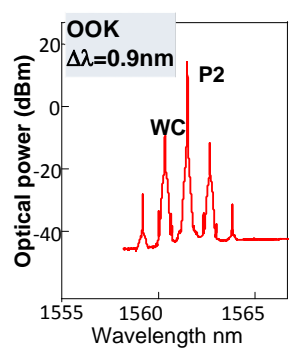

(b)

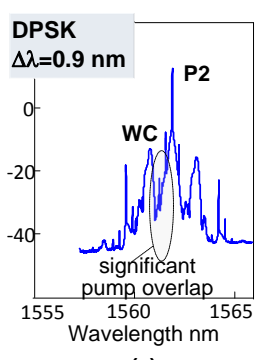

(c)

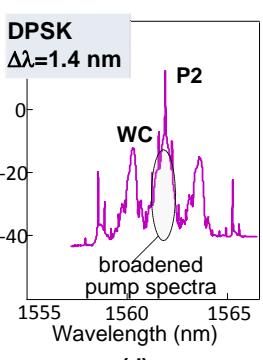

(d)

Figure 3. (a) DPSK spectrum, pump detuning $\Delta \lambda=1.4 \mathrm{~nm}$; zoom on conversion products for (b) OOK with $\Delta \lambda=0.9 \mathrm{~nm}$, DPSK with (c) $\Delta \lambda=0.9 \mathrm{~nm}$, and (d) $\Delta \lambda=1.4 \mathrm{~nm}$.

Conversion efficiency is highest when keeping detuning between pump1 and the input signal as small as possible. The detuning $\Delta \lambda$ between $\lambda_{\text {Sig }}$ and $\lambda_{\text {pump }}$ is set to $0.9 \mathrm{~nm}$ for OOK, as in [12]. Maintaining adequate separation between the second pump and the signal conjugate (spacing equal to $\Delta \lambda$ ) constrains proximity: the tunable output filter must remove all but the desired conjugate signal. The DPSK signal has significantly higher sidelobes than the OOK signal, requiring greater separation. In Figure 3a we present the entire output spectrum of the XN-SOA for an input DPSK signal and detuning of $\Delta \lambda=1.4 \mathrm{~nm}$ when converting input $\lambda_{1}=1548.5 \mathrm{~nm}$ to output $\lambda_{15}=1560.5 \mathrm{~nm}$. We see good separation between pump2 and the wavelength converted (WC) signal. Note that in the DPSK case, the 
pump2 has acquired a spectrum considerably more broadened by beating with the DPSK signal than the OOK case (constrast with Figure $3 b$ ). In Figure 3b, c, and d we zoom in on pump2 and WC for three separate cases. In Figure $3 b$ we have an OOK signal and in Figure $3 c$ DPSK, both for $\Delta \lambda=0.9 \mathrm{~nm}$. While both operate at $40 \mathrm{~Gb} / \mathrm{s}$, for OOK the WC signal and the pump are well separated, while the DPSK WC signal has significant overlap with pump2. Even when using tight filtering of the WC signal, the power from the sidelobes of pump2 deteriorate the optical signal to noise ratio. Sweeping wavelength separation, we found that a separation of $\Delta \lambda=1.4 \mathrm{~nm}$ (shown in Figure 3d) optimized conversion efficiency.

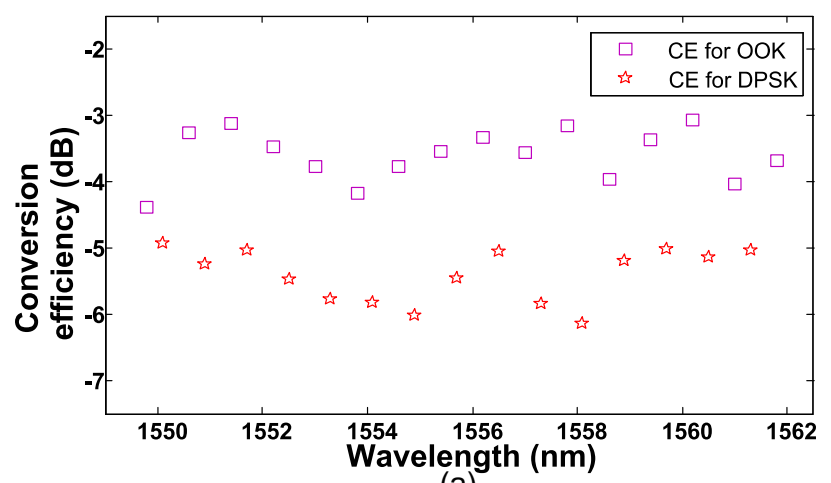

(a)

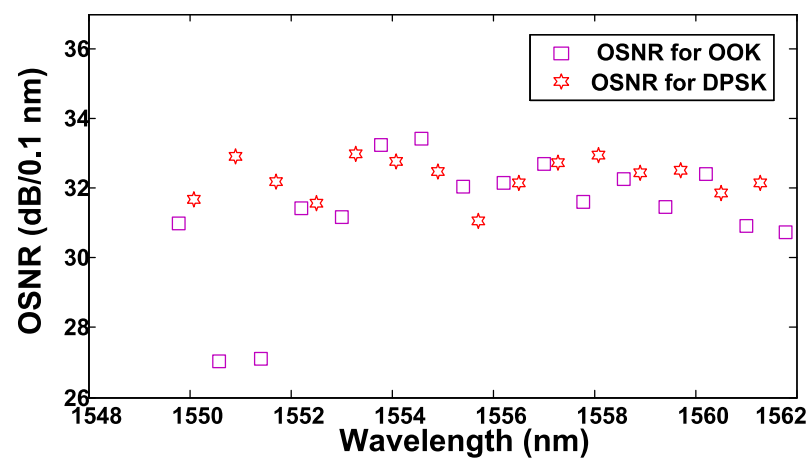

(b)



(c)

Figure 4. (a) Conversion efficiency $(\mathrm{dB})$, input at $\lambda_{1}$, (b) converted signal optical signal-to-noise ratio (OSNR) $(\mathrm{dB} / 0.1 \mathrm{~nm})$ for OOK and DPSK, and (c) spectrum for OOK conversion from $\lambda_{1}$ to $\lambda_{3}$.

The measured conversion efficiency versus the wavelength of the converted signal is depicted in Figure $4 \mathrm{a}$. We achieve conversion efficiency greater than $-4.4 \mathrm{~dB}$ for OOK and greater than $-6.1 \mathrm{~dB}$ for 
DPSK within the measured conversion range of $12 \mathrm{~nm}$. The OSNR, as measured at point C of Figure 2, is defined as the ratio of the signal peak power and the noise power level. The conversion efficiency depends on the detuning values between the signal and the closest pump as demonstrated in [11]. Therefore, the conversion efficiency value for DPSK is lower than for OOK because the detuning between the signal and the closest pump is larger for DPSK.

We express all the OSNR values in the reference resolution bandwidth of $0.1 \mathrm{~nm}$ and present measured results in Figure 4b. The measured OSNR at the output of the XN-SOA is greater than $27 \mathrm{~dB}$ for OOK and greater than $30 \mathrm{~dB}$ for DPSK throughout the wavelength range. We notice that for OOK, the OSNR decreases for output wavelengths $\lambda_{3}=1550.6 \mathrm{~nm}$ and $\lambda_{4}=1551.4 \mathrm{~nm}$. This results from a second type of constraint on placement of signal and pump. Consider conversion when input and output signals are closely packed. Unlike the example in Figure 3a, pump1 must be set at a wavelength lower than input $\lambda_{1}$ to leave space for the output signal at a wavelength just above that of the input signal. When converting to $\lambda_{3}$, the output wavelength is nested between the two pumps as seen in Figure 4c, leading to OSNR degradation. It appears that the broadened pump1 deteriorates the OSNR. Clearly optimizing the position of the pump wavelengths (left/right of input and/or output signals) could lead to further improvement, while requiring slightly more pump wavelengths.

Previous studies $[8,10]$ demonstrated conversion efficiency below $-8 \mathrm{~dB}$ : $-10 \mathrm{~dB}$ for OOK and $-11.5 \mathrm{~dB}$ for DPSK in [10] and $-8 \mathrm{~dB}$ in [8]. Pump powers were high, $7 \mathrm{dBm}$ in [8] and $P_{P_{1}}=10.5 \mathrm{dBm}$ and $P_{P_{2}}=6.5 \mathrm{dBm}$ for OOK and $P_{P_{1}}=11 \mathrm{dBm}$ and $P_{P_{2}}=7 \mathrm{dBm}$ for DPSK in [10]. We achieve higher conversion efficiency ( $-3.6 \mathrm{~dB}$ in average for OOK and $-5.4 \mathrm{~dB}$ for DPSK) with an OSNR of $\sim 31.3 \mathrm{~dB} / 0.1 \mathrm{~nm}$ for OOK and $\sim 32.1 \mathrm{~dB} / 0.1 \mathrm{~nm}$ for DPSK on average at pump powers below $1 \mathrm{dBm}$. The improvement is most likely due to the smaller detuning between the signal and first pump in our experiments. In [10] a detuning of $6.6 \mathrm{~nm}$ was used in order to achieve polarization independence, but at the cost of a bit error floor and extremely limited tuning range. In [8] the detuning was $1.6 \mathrm{~nm}$, but a different SOA was used and operating points were chosen to optimize performance in a cascade of 10 wavelength conversions; tuning range was not examined and only a single pump was used.

The conversion efficiency for 48 separate experiments over $12 \mathrm{~nm}$ tuning range is reported in Table 1 for OOK and DPSK. We consider 16 wavelengths for OOK $\left(\lambda_{S i g}=1549 \mathrm{~nm}\right.$ for original signal and $\lambda_{W C}$ from $1549.8 \mathrm{~nm}$ to $1561 \mathrm{~nm}$ for the converted signals) and for DPSK ( $\lambda_{S i g}=1548.5 \mathrm{~nm}$ for original signal and $\lambda_{W C}$ from $1550.1 \mathrm{~nm}$ to $1561.3 \mathrm{~nm}$ for the converted signals). The conversion efficiency for the converted signals shown in Figure $3 \mathrm{a}$ is reported in the first row of Table 1. From the first row of Table 1, the conversion efficiency fluctuates by $1.3 \mathrm{~dB}$ for OOK and $1.2 \mathrm{~dB}$ for DPSK with best case: $-3.1 \mathrm{~dB}$ for OOK and $-4.9 \mathrm{~dB}$ for DPSK; and worst case: $-4.4 \mathrm{~dB}$ for OOK and $-6.1 \mathrm{~dB}$ for DPSK.

We have an average conversion efficiency of $-3.5 \mathrm{~dB}$ for OOK (and $-5.6 \mathrm{~dB}$ for DPSK) for $\lambda_{8}$ and $-3.8 \mathrm{~dB}$ for OOK (and $-5.7 \mathrm{~dB}$ for DPSK) for $\lambda_{16}$, we expect that approximate values are achievable for the other wavelengths. For the 48 performed measurements, we achieve high conversion efficiency with an average value of $-3.6 \mathrm{~dB}$ for OOK and $-5.6 \mathrm{~dB}$ for DPSK. The maximum/minimum values for $\mathrm{CE}$ are $-2.6 /-4.9 \mathrm{~dB}$ and $-5.1 /-6.7 \mathrm{~dB}$, for OOK and DPSK, respectively. The standard deviation is $0.2 \mathrm{~dB}$ for both OOK and DPSK. We notice that the values for conversion efficiency do not degrade with a wider conversion range. Using 16 pump wavelengths, we could theoretically cover an even wider tuning range $(12 \mathrm{~nm})$ to achieve 240 conversions over 16 channels. The conversion efficiency depends 
on the detuning values between the signal and the closest pump as demonstrated in [12]. Therefore, the conversion efficiency value for DPSK is lower than for OOK because the detuning between the signal and the closest pump is larger for DPSK.

Table 1. Absolute value of conversion efficiency.

\begin{tabular}{|c|c|c|c|c|c|c|c|c|c|c|c|c|c|c|c|c|c|}
\hline & & $\lambda_{1}$ & $\lambda_{2}$ & $\lambda_{3}$ & $\lambda_{4}$ & $\lambda_{5}$ & $\lambda_{6}$ & $\lambda_{7}$ & $\lambda_{8}$ & $\lambda_{9}$ & $\lambda_{10}$ & $\lambda_{11}$ & $\lambda_{12}$ & $\lambda_{13}$ & $\lambda_{14}$ & $\lambda_{15}$ & $\lambda_{16}$ \\
\hline \multirow{2}{*}{$\lambda_{1}$} & OOK & & 4.4 & 3.3 & 3.1 & 3.5 & 3.8 & 4.2 & 3.8 & 3.5 & 3.3 & 3.6 & 3.2 & 4.0 & 3.4 & 3.1 & 4.0 \\
\hline & DPSK & & 4.9 & 5.3 & 5.8 & 5.8 & 6.0 & 5.5 & 5.1 & 5.9 & 6.1 & 5.2 & 5.0 & 5.1 & 5.0 & 5.8 & 5.8 \\
\hline \multirow{2}{*}{$\lambda_{8}$} & OOK & 5.0 & 4.5 & 4.3 & 4.0 & 3.5 & 3.2 & 2.8 & & 3.3 & 3.7 & 3.4 & 3.6 & 3.7 & 3.5 & 3.4 & 3.4 \\
\hline & DPSK & 5.3 & 5.2 & 5.4 & 6.0 & 5.4 & 6.1 & 5.9 & & 6.0 & 6.0 & 5.8 & 5.8 & 5.2 & 5.1 & 5.1 & 5.5 \\
\hline \multirow{2}{*}{$\lambda_{16}$} & OOK & 5.3 & 5.1 & 4.7 & 4.2 & 4.0 & 4.1 & 3.8 & 4.0 & 3.5 & 3.6 & 3.7 & 3.5 & 3.1 & 2.9 & 2.6 & \\
\hline & DPSK & 6.7 & 6.6 & 6.4 & 6.5 & 6.2 & 6.0 & 5.9 & 5.5 & 5.7 & 5.4 & 5.2 & 5.3 & 5.1 & 5.0 & 5.0 & \\
\hline
\end{tabular}
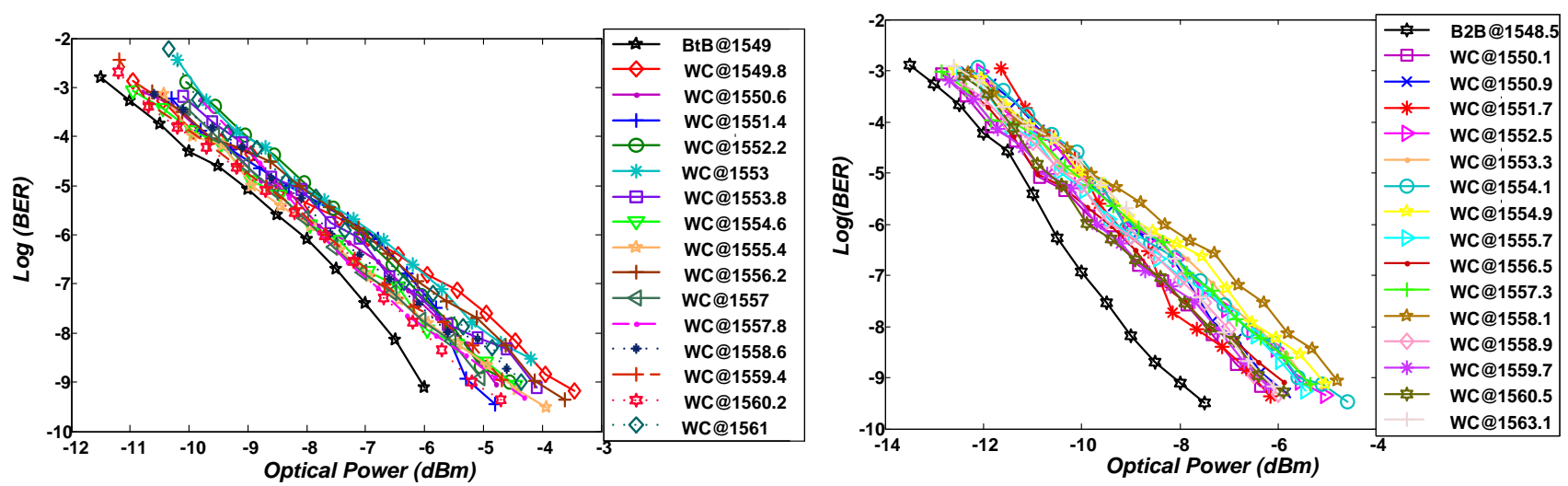

Figure 5. Bit error rate $v s$. receiver input power for 16 wavelength conversions: (a) OOK for conversion range from $0.8 \mathrm{~nm}$ to $12 \mathrm{~nm}$, (b) DPSK for conversion range from $1.6 \mathrm{~nm}$ to $12.8 \mathrm{~nm}$.

BER measurements are performed over a $12 \mathrm{~nm}$ conversion range covering 16 channels with $100 \mathrm{GHz}$ spacing as shown in Figure 4. Results for BER vs. received optical power (measured at point D of Figure 2) are presented in Figure 5, with OOK reported in Figure 5a and DPSK in Figure 5b. Due to the copolarized dual pump configuration, the system is optimized for each converted wavelength using the polarization controllers to maximize the pump alignment. The power penalty at BER of $10^{-9}$ is used as a measure of the system performance degradation by the wavelength conversion process. Moderate power penalty is measured as the original signal is successively converted into the 16 new wavelengths for both OOK and DPSK (lower than $2 \mathrm{~dB}$ for OOK and lower than $3 \mathrm{~dB}$ for DPSK). Comparing Figure 4a with BER results, we see the same trends from conversion efficiency repeated in the BER results. 
Finally, we examine OSNR for conversion to $\lambda_{9}\left(\lambda_{W C}=1555.4 \mathrm{~nm}\right.$ for OOK, $\lambda_{W C}=1555.7 \mathrm{~nm}$ for DPSK). We report measured output OSNR versus the input OSNR in Figure 6a, observing a linear relationship between the input and output OSNR as expected. We also study the impact of the input OSNR degradation on the conversion efficiency. We observe a conversion efficiency degradation with decreasing input OSNR that is less than $2 \mathrm{~dB}$ for OOK and less than $1 \mathrm{~dB}$ for DPSK, as shown in Figure $6 b$.
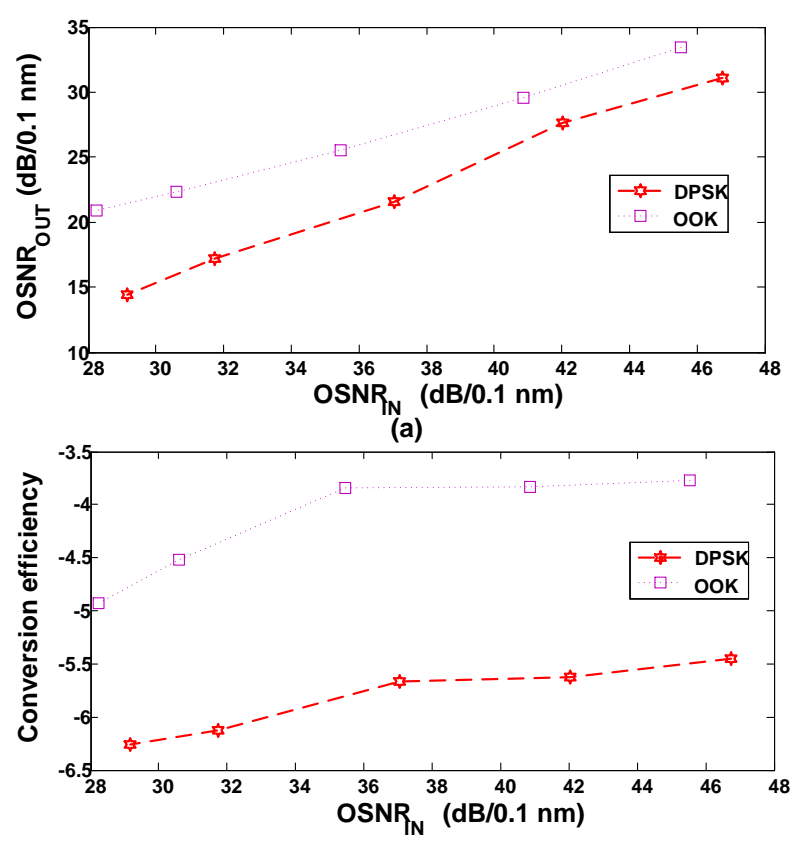

(b)

Figure 6. (a) Output OSNR vs. input OSNR, and (b) conversion efficiency vs. input OSNR.

\section{Conclusion}

In summary, we have proposed and experimentally demonstrated an all-optical modulation-format and high bit-rate transparent wavelength conversion at the $40 \mathrm{Gbit} / \mathrm{s}$ using a QD-MLL as a dual pump source and FWM in an XN-SOA. A performance comparison of the OOK and DPSK signals using the proposed wavelength converter is reported. We reported error-free signal conversion over 16 channels with $100 \mathrm{GHz}$ spacing for both OOK and DPSK with moderate power penalty (less than $2 \mathrm{~dB}$ for OOK and than $3 \mathrm{~dB}$ for DPSK). The high conversion efficiency values were obtained at relatively low input pump powers over a wide wavelength range. We demonstrated that optical SOA wavelength converters, transparent to bit rates and modulation formats, are very good and viable solutions for next generation optical communication networks. The significant sidelobes of the DPSK spectrum (relative to OOK), leading to a modest power penalty $(1 \mathrm{~dB})$ for DPSK relative to OOK.

\section{Acknowledgements}

This research was supported by the Natural Sciences and Engineering Research Council of Canada (NSERC) and CMC Microsystems. The authors would like to thank A. Shen, F. Lelarge, F. Pommereau 
and D. Make from III-V Lab, a joint lab of Alcatel-Lucent Bell Labs France, Thales Research and Technology and CEA Leti.

\section{Author Contributions}

Y. BenMSallem was responsible for running the experiment and analyzing the data. C.S. Park contributed extensively to planning the experimental setup, as well as assisting in conducting the experiment. LaRochelle and Rusch were responsible for defining the research objectives, interpreting experimental results and putting results in context. All authors contributed to the writing of the paper.

\section{Conflicts of Interest}

The authors declare no conflict of interest.

\section{References}

1. Winzer, P.J.; Essiambre, R.-J. Advanced modulation formats for high-capacity optical transport networks. IEEE/OSA J. Lightw. Technol. 2006, 24, 4711-4728.

2. Murthy, C.S.R.; Gurusamy, M. WDM Optical Networks: Concepts, Design, and Algorithms; Prentice Hall PTR Englewood Cliffs: Upper Saddle River, NJ, USA, 2002.

3. Xue, F.; Pan, Z.; Bansal, Y.; Cao, J.; Jeon, M.; Okamoto, K.; Kamei, S.; Akella, V.; Yoo, S. End-to-end contention resolution schemes for an optical packet switching network with enhanced edge routers. IEEE/OSA J. Lightw. Technol. 2003, 21, 2595-2604.

4. De Laat, M.; Duijn, R.; Pluk, E.; van den Hoven, G.; Urban, P.; de Waardt, H. Flexpon: A hybrid TDM/WDM network enabling dynamic bandwidth reconfiguration using wavelength routing. In Proceedings of the 35th European Conference on Optical Communication, Vienna, Austria, 20-24 September 2009.

5. Gripp, J.; Simsarian, J.; LeGrange, J.; Bernasconi, P.; Neilson, D. Architectures, components, and subsystems for future optical packet switches. IEEE J. Sel. Top. Quantum Electron. 2010, $16,1394-1404$.

6. Diez, S.; Schmidt, C.; Ludwig, R.; Weber, H.; Obermann, K.; Kindt, S.; Koltchanov, I.; Petermann, K. Four-wave mixing in semiconductor optical amplifiers for frequency conversion and fast optical switching. IEEE J. Sel. Top. Quantum Electron. 1997, 3, 1131-1145.

7. Yoo, S.B. Wavelength conversion technologies for WDM network applications. IEEE/OSA J. Lightw. Technol. 1996, 14, 955-966.

8. Li, Z.; Dong, Y.; Mo, J.; Wang, Y.; Lu, C. Cascaded all-optical wavelength conversion for RZ-DPSK signal based on four-wave mixing in semiconductor optical amplifier. Photon. Technol. Lett. 2004, 16, 1685-1687.

9. Lacey, J.P.; Summerfield, M.A.; Madden, S. Tunability of polarization-insensitive wavelength converters based on four-wave mixing in semiconductor optical amplifiers. IEEE/OSA J. Lightw. Technol. 1998, 16, 2419-2427. 
10. Contestabile, G.; Banchi, L.; Ciaramella, E.; Presi, M. Investigation of transparency of FWM in SOA to advanced modulation formats involving intensity, phase, and polarization multiplexing. IEEE/OSA J. Lightw. Technol. 2009, 27, 4256-4261.

11. Contestabile, G.; Yoshida, Y.; Maruta, A.; Kitayama, K. Ultra-broad band, low power, highly efficient coherent wavelength conversion in quantum dot SOA. Opt. Express 2012, 20, 27902-27907.

12. M'Sallem, Y.B.; Shen, A.; Lelarge, F.; Pommereau, F.; Make, D.; LaRochelle, S.; Rusch, L.A. Quantum-dash mode-locked lasers for tunable wavelength conversion on a $100 \mathrm{GHz}$ frequency grid. J. Opt. Commun. Netw. 2012, 4, A69-A76.

13. Lee, S.-L.; Chien, C.-Y.; Tsao, H.-W.; Wu, J. Practical considerations of using tunable lasers for packet routing in multiwavelength optical networks. In Proceedings of the International Conference on Parallel Processing Workshops, Kaohsiung, Taiwan, 6-9 October 2003; pp. 325-331.

14. Coldren, L.; Fish, G.; Akulova, Y.; Barton, J.; Johansson, L.; Coldren, C. Tunable semiconductor lasers: A tutorial. IEEE/OSA J. Lightw. Technol. 2004, 22, 193-202.

15. Shi, K.; Anandarajah, P.; Reid, D.; Smyth, F.; Barry, L.; Yu, Y. SG-DBR tunable laser linewidth and its impact on advanced modulation format transmission. In Proceedings of the IEEE European Conference on Lasers and Electro-Optics and the European Quantum Electronics Conference, Munich, Germany, 14-19 June 2009.

16. Duan, G.-H.; Shen, A.; Akrout, A.; Dijk, F.V.; Lelarge, F.; Pommereau, F.; LeGouezigou, O.; Provost, J.-G.; Gariah, H.; Blache, F.; et al. High performance InP-based quantum dash semiconductor mode-locked lasers for optical communications. Bell Labs Tech. J. 2009, 14, 63-84.

17. Gnauck, A.H.; Winzer, P.J. Optical phase-shift-keyed transmission. IEEE/OSA J. Lightw. Technol. 2005, 23, 115-130.

18. Xu, C.; Liu, X.; Wei, X. Differential phase-shift keying for high spectral efficiency optical transmissions. IEEE J. Sel. Top. Quantum Electron. 2004, 10, 281-293.

19. Gupta, S.; Calabretta, N.; Contestabile, G.; Ciaramella, E.; Gangopadhyay, R. Experimental characterization of SOA-based wavelength converters for DPSK signals. In Proceedings of the First International Conference on Communications and Networking in China, Beijing, China, 25-27 October 2006; pp. 1-5.

20. Okoshi, T.; Kikuchi, K.; Nakayama, A. Novel method for high resolution measurement of laser output spectrum. Electron. Lett. 1980, 16, 630-631.

21. Tomkos, I.; Zacharopoulos, I.; Roditi, E.; Syvridis, D.; Girardin, F.; Occhi, L.; Uskov, A. Highly performing wavelength converter based on dual pump wave mixing in semiconductor optical amplifier. Proc. IEEE Lasers Electro-Optics Soc. Annu. Meet. 1998, 1, 71-72.

22. Hong, Y.; Bandyopadhyay, S.; Spencer, P.; Shore, K. Polarization-independent optical spectral inversion without frequency shift using a single semiconductor optical amplifier. IEEE J. Quantum Electron. 2003, 39, 1123-1128. 
23. Otsubo, K.; Tanaka, S.; Tomabechi, S.; Morito, K.; Kuwatsuka, H. High efficiency, wide range and completely transparent wavelength conversion method using replicas generated by dual pump nearly-degenerated four-wave mixing in a mach-zehnder interferometer SOA. In Proceendings of the Optical Fiber Communication Conference, and National Fiber Optic Engineers Conference, Anaheim, CA, USA, 5-10 March 2006.

24. D’ottavi, A.; Girardin, F.; Graziani, L.; Martelli, F.; Spano, P.; Mecozzi, A.; Scotti, S.; Dall'Ara, R.; Eckner, J.; Guekos, G. Four-wave mixing in semiconductor optical amplifiers: A practical tool for wavelength conversion. IEEE J. Sel. Top. Quantum Electron. 1997, 3, 522-528.

(c) 2015 by the authors; licensee MDPI, Basel, Switzerland. This article is an open access article distributed under the terms and conditions of the Creative Commons Attribution license (http://creativecommons.org/licenses/by/4.0/). 